\title{
SPATIAL ORIENTATION OF GALAXIES IN SUPERCLUSTER S[195+027+0022]
}

\author{
J. R. Malla, B. Aryal and W. Saurer
}

Journal of Nepal Physical Society

Volume 5, Issue 1, October 2019

ISSN: $2392-473 X$

Editors:

Dr. Vinaya Kumar Jha

Dr. Binod Adhikari

Dr. Kapil Adhikari

JNPS, 5 (1), 14-18 (2019)

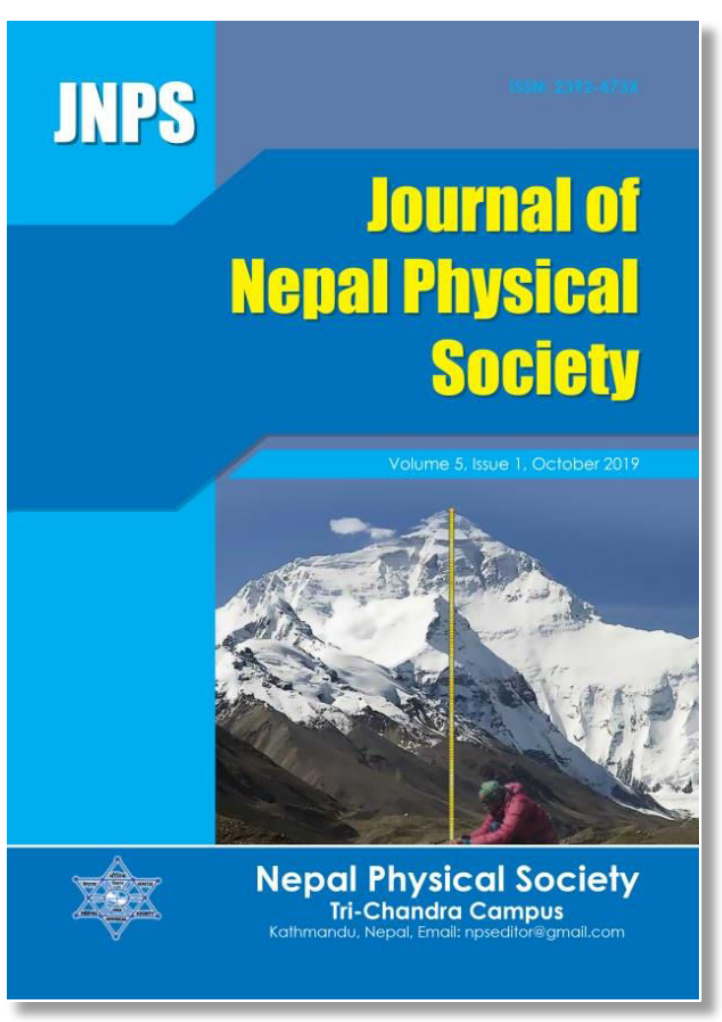

Published by:

Nepal Physical Society

P.O. Box: 2934

Tri-Chandra Campus

Kathmandu, Nepal

Email:npseditor@gmail.com 


\title{
SPATIAL ORIENTATION OF GALAXIES IN SUPERCLUSTER S[195+027+0022]
}

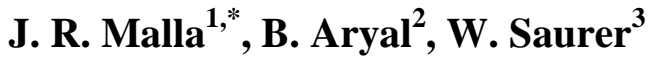 \\ ${ }^{1}$ Amrit Science Campus, TU, Kathmandu, Nepal \\ ${ }^{2}$ Central Department of Physics, TU, Kirtipur, Kathmandu, Nepal \\ ${ }^{3}$ Institute of Astro-particle Physics, Innsbruck University, Austria \\ "Corresponding Email: janak_malla@yahoo.com
}

\begin{abstract}
We present a study of spin vector orientation of 1198 SDSS (Sloan Digital Sky Survey) galaxies in Supercluster S[195+027+0022] having redshift 0.07 to 0.09 . The databases of these galaxies are taken from SDSS (Sloan Digital Sky Survey) $7^{\text {th }}$ and $9^{\text {th }}$ data release. We have converted two dimensional data to three dimensional by Godlowskian Transformation using position angle-inclination angle method. We intend to find non-random effects in the spatial orientation of galaxies in Supercluster. No preferred alignment of angular momentum vectors (spin vector) is noticed, supporting Hierarchy model of galaxy formation.
\end{abstract}

Keywords: SDSS, 2D-FGRS, Filament, SV, Supercluser, Redshift, Hump, Dip.

\section{INTRODUCTION}

Modern cosmology is based on two fundamental assumptions: First, the dominant interaction on cosmological scales is gravity, and second, the cosmological principle is a good approximation to the universe. The cosmological principle states that the universe, smoothed over large enough scales, is essentially homogeneous and isotropic. 'Homogeneity' has the intuitive meaning that at a given time the universe looks the same everywhere, and 'isotropy' refers to the fact that for any observer moving with the local matter the universe looks (locally) the same in all directions. Von Weizsacker and Gamow [24] verified that the observed rotation of the galaxies is important for cosmology: the fact that the galaxies rotate may be clue to the physical conditions under which these systems formed. In the instability picture, one imagines that large irregularity like galaxies grew under the in influence of gravity from small imperfections in the early Universe [15-17]. In this picture one must abandon the idea that the angular momentum of the galaxies was given as the initial value, or developed in some sort of primeval turbulence (as was proposed by Von Weizsaker [24] and Gamow [14], for otherwise the galaxies would have formed too soon [21]. On the other hand, huge low red shift galaxy surveys such as the2-degree field galaxy red shift survey [8] and the Sloan Digital Sky Survey [25] have convinced most cosmologists that not only isotropy but also homogeneity is in fact a reasonable assumption for the universe. The Universe is homogeneous and isotropic on scales larger than $100 \mathrm{Mpc}$, but on smaller scales we observe huge deviations from the mean density in the form of galaxies, galaxy clusters, and the cosmic web being made of sheets and filaments of galaxies. How do structures grow in the universe and how can we describe them? The most accepted view on the formation and evolution of large scale structure is that it was formed as a consequence of the growth of primordial fluctuations by gravitational instability. In the current favored model, smaller structures collapse first and are later incorporated in larger collapsing structures in a bottom-up scenario that provides a natural explanation for the formation of galaxies, clusters, filaments and Superclusters.

There are three predictions about the spatial orientation of spin vectors of galaxies. These are the 'pancake model', the 'hierarchy model,' and the 'primordial vorticity theory.' The 'pancake model' [9-11] predicts that the spin vectors (SVs hereafter) of galaxies tend to lie within the cluster plane. According to the 'hierarchy model' [22], the directions of the SVs should be distributed randomly. The 'Primordial Vorticity Theory' [20, 23] predicts that the spin vectors of galaxies are 
distributed primarily perpendicular to the cluster plane.

In this paper, we analyze spatial orientation of angular momentum vectors of 1,198 galaxies in the SDSS supercluster S[195+027+0022]. The methods and results are described in the following chapters.

\section{Godlowskian Transformation}

The three dimensional orientation of the SV of a galaxy is characterized by two angles: the polar angle $(\theta)$ between the galactic SV and a reference plane (here equatorial plane), and the azimuthal angle $(\phi)$ between the projection of a galactic SV on to this reference plane and the $\mathrm{X}$-axis within this plane. The detail derivations of the expressions of the angles $\theta$ and $\phi$ are given in Flin \& Godłowski $[12,13]$. When using equatorial coordinate system as reference, then $\theta$ and $\phi$ can be obtained from measurable quantities as follows:

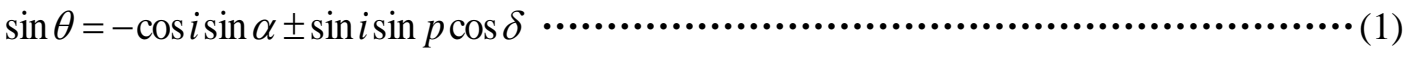

$$
\begin{aligned}
& \sin \phi=(\cos \theta)^{-1}[-\cos i \cos \delta \sin \alpha+\sin i(\mu \sin p \sin \delta \sin \alpha \mu \cos p \cos \alpha)] \cdots \ldots \ldots \ldots \ldots \ldots \ldots \ldots . .(2)
\end{aligned}
$$

Where, $\mathrm{i}$ is the inclination angle, the angle between the normal to the galaxy plane and the observer's line-of-sight, $\alpha$ - is right ascension, $\delta$ is declination and $\mathrm{p}$ is position angle. The inclination angle can be computed from the formula

$$
\cos ^{2} i=\frac{\left(q^{2}-q^{*^{2}}\right)}{\left(1-q^{*^{2}}\right)} \cdots \cdots \cdots
$$

This expression is valid for oblate spheroids [18]. Here, $q$ and $q^{*}$ represent the measured axial ratio (b/a) and the intrinsic flatness of the galaxy, respectively. Hiedmann et al. [19] showed that the values of $q^{*}$ range from 0.083 for $\mathrm{Sd}$ spirals to 0.33 for ellipticals. For the galaxies with unknown morphology $q^{*}=0.20$ is assumed [18].

\section{METHOD OF ANALYSIS}

Here we describe the procedure for the removal the selection effects to obtain the isotropic distributions for both $\theta$ and $\phi$ as given by Aryal \& Saurer [1-7]. Theoretically, the isotropic distribution curve for polar angle is cosine and that for azimuthal angle is the average distribution curve, with the restriction that the database is free from selection effect. Aryal \& Saurer [7] concluded that any selections imposed on the database may cause severe changes in the shapes of the expected isotropic distribution curves. In their method, a true spatial distribution of the galaxy rotation axis is assumed to be isotropic. Then, due to the projection effects, $i$ can be distributed as $\_\sin i, B$ can be distributed ${ }_{\sim} \cos B$, the variables $\alpha$ and $p$ can be distributed randomly, and the equation $(1,2)$ can be used to calculate the corresponding values of polar $(\theta)$ and azimuthal $(\phi)$. We run simulations in order to define expected isotropic distribution curves for both the $\theta$ and $\phi$ distributions. The isotropic distribution curves are based on simulations including $10^{7}$ virtual galaxies.
At first we observed the distributions of $\alpha, \delta$, p and $i$ for the galaxies in our samples and distributed by creating $10^{7}$ virtual galaxies for respective parameters. We use these numbers to make input file and the expected distribution by running simulation in MATLAB 15.0.

Our observations (real observed data set) are compared with the isotropic distribution curves (obtained from simulation) in both $\theta$ and $\phi$ distributions. For this comparison we use four different statistical tests: chi-square probability, First order Fourier Coefficient $\left(\Delta_{11} / \sigma\left(\Delta_{11}\right)\right)$, Fourier Probability $\left(\mathrm{P}>\Delta_{1}\right)$ and auto correlation-test $(\mathrm{C} / \mathrm{C}(\sigma))$.

\section{RESULTS \& DISCUSSION}

Any deviation from expected isotropic distribution will be tested using four statistical parameters, namely chi-square probability $\left(\mathrm{P}>\chi^{2}\right)$, autocorrelation coefficient $(\mathrm{C} / \mathrm{C}(\sigma))$, first order Fourier coefficient $\left(\Delta_{11} / \sigma\left(\Delta_{11}\right)\right)$ and first order Fourier probability $\left(\mathrm{P}>\Delta_{1}\right)$. For anisotropy, the limit of chi-square probability $\mathrm{P}\left(>\chi^{2}\right)$ is $<0.050$, auto correlation coefficient $(\mathrm{C} / \mathrm{C}(\sigma))$ is $>1.0$, first order Fourier coefficient $\left(\Delta_{11} / \sigma\left(\Delta_{11}\right)\right)$ is $>1.5$ and Fourier probability $\mathrm{P}\left(>\Delta_{1}\right)$ is $<0.150$ respectively. Any 'hump' (more solutions than the expected) or 'dip' (less solution than the expected) in the histogram will be discussed as a local effect in the samples. The statistics for the polar and azimuthal angle distributions is given in Table 1 . In the statistics of $\theta$, a negative value of first order Fourier coefficient suggests that the spin vectors of galaxies tend to be oriented perpendicular with respect to the equatorial coordinate system. Similarly, a positive value of first order Fourier coefficient suggests that the spin vectors of galaxies tend to be oriented parallel with respect to the equatorial coordinate system. Whereas, in the statistics of $\phi$, a positive 
$\left(\Delta_{11} / \sigma\left(\Delta_{11}\right)\right.$ with significant value suggests that the spin vector projections of galaxies tend to point radially with respect to the center of the equatorial coordinate system. Similarly, a significant negative value of $\left(\Delta_{11} / \sigma\left(\Delta_{11}\right)\right.$ implies that the spin vector projection of galaxies tend to orient tangentially with respect to the equatorial coordinate system.

In addition to the statistical tests, we also study the 'humps' and 'dips' in the polar and azimuthal angle distributions. The solid curve, in the histogram of the $\theta$-distribution, represents the expected isotropic distribution where as dashed curve is the cosine distribution. The solid circles with $\pm 1 \sigma$ error bars represent the observed distribution. The shaded portion represents the range $0^{\circ}<\theta<45^{\circ}$. A hump (or dip) in the smaller $\theta(\theta<450)$ suggests that the spin vectors of galaxies tend to orient parallel (or perpendicular) with respect to the equatorial coordinate system. Similarly, a hump (or dip) in the larger $\theta\left(\theta>45^{\circ}\right)$ suggests that the spin vectors of galaxies tend to orient perpendicular with respect to the equatorial coordinate system. In figure 1 , there is a hump on polar angle distribution at $\theta=46$. This hump is due to 13 more expected solution than observed solutions in the range $\theta>45^{\circ}$.

Similarly, a hump (or dip) in the larger $\phi$ indicates that the spin vectors of galaxies tend to be oriented perpendicular with respect to the equatorial coordinate system. In the histogram of the $\phi$ distribution, solid curve represents the expected isotropic distribution whereas dashed curve is the average distribution. The solid circles with $\pm 1 \sigma$ error bars represent the observed distribution. The shaded portion represents the range $-45^{\circ}<\phi<+45^{\circ}$. The humps and dips in the histograms of $\phi$ distribution are not so easy to interpret as compared to $\phi$-distributions. It is because the range of $\phi$ is $90^{\circ}$ to $+90^{\circ}$. In the histogram of the $\phi$-distribution, $\phi=0^{\circ}$ means spin vector projections tend to point radially towards the center of the equatorial coordinate system. A hump in the middle (central eight bins) of the histogram suggests that the spin vector projections of galaxies tend to point towards the center of the chosen co-ordinate system. Similarly, a hump at first four and last four bins indicates that the spin vectors projections of galaxies tend to be oriented tangentially with respect to the chosen reference co-ordinate system. A hump in the middle (central eight bins) of the plot suggests that the spin vector projections of galaxies tend to point towards the center of the chosen co-ordinate system.
The statistics for the $\theta$-distribution of galaxies of Supercluster $S(195+027+0022)$ is shown in the Table 1. The statistics for the polar angle distribution in this sample shows that the value of chi-square probability $\left(\mathrm{P}\left(>\chi^{2}\right)\right)$ to be 0.0 i.e., 0 (Smaller than the significant level 0.050 i.e., $5.0 \%)$. The auto-correlation coefficient $(\mathrm{C} / \mathrm{C}(\sigma))$ is found to be 3.9 (greater than $1 \sigma$ limit). The first order Fourier coefficient $\left(\Delta_{11} / \sigma\left(\Delta_{11}\right)\right)$ is found to be 0.0 (smaller than $1.5 \sigma$ the limit). The first order Fourier probability $(\mathrm{P}>(\Delta 1))$ is found to be 0.084 (Smaller than 0.15 i.e.,15\%). Except first order fourier coefficient, other statistical tests suggest anisotropy. Isotropy in the first order fourier coefficient test suggests that the direction of departure from isotropy.

The statistics of the $\varphi$-distribution of galaxies of Supercluster $S[195+027+0022]$ is shown in the Table 1. The statistics for the azimuthal angle distribution in this sample shows that the value of chi-square probability $\left(\mathrm{P}\left(>\chi^{2}\right)\right)$ to be 0.0 i.e., 0 (Smaller than the significant level 0.050 i.e., $5.0 \%)$. The auto-correlation coefficient $(\mathrm{C} / \mathrm{C}(\sigma))$ is found to be 8.7 (greater than $1 \sigma$ limit). The first order Fourier coefficient $\left(\Delta_{11} / \sigma\left(\Delta_{11}\right)\right)$ is found to be 0 (smaller than $1.5 \sigma$ the limit). The first order Fourier probability $(\mathrm{P}>(\Delta 1))$ is found to be 0.0 (Smaller than 0.15 i.e.,15\%). Except first order Fourier coefficient, other statistical tests suggest anisotropy. Isotropy in the first order Fourier coefficient test suggests that the direction of departure from isotropy.

Table 1: Statistics of the polar and azimuthal angle distributions of galaxies in the Supercluster $\mathrm{S}(195+027+0022)$. The first column represents the statistics used, $\mathrm{P}\left(>\chi^{2}\right)$ represents the chi-square probability (second row). Similarly, $\mathrm{C} / \mathrm{C}(\sigma)$ represents the auto-correlation coefficient(third row). The last two rows give the first order Fourier coefficient $\left(\Delta_{11} / \sigma\left(\Delta_{11}\right)\right.$ and first order Fourier probability $\mathrm{P}\left(>\Delta_{1}\right)$.

\begin{tabular}{|l|c|c|}
\hline \multicolumn{1}{|c|}{ Statistics } & $\begin{array}{c}\text { Polar } \\
\text { angle }(\boldsymbol{\theta})\end{array}$ & $\begin{array}{c}\text { Azimuthal } \\
\text { angle }(\phi)\end{array}$ \\
\hline $\mathrm{P}\left(>\chi^{2}\right)$ & 0.000 & 0.000 \\
\hline $\mathrm{C} / \mathrm{C}(\sigma)$ & 3.9 & 8.7 \\
\hline$\Delta 11 / \sigma(\Delta 11)$ & 0.0 & 0.0 \\
\hline $\mathrm{P}(>\Delta 1)$ & 0.084 & 0.000 \\
\hline
\end{tabular}



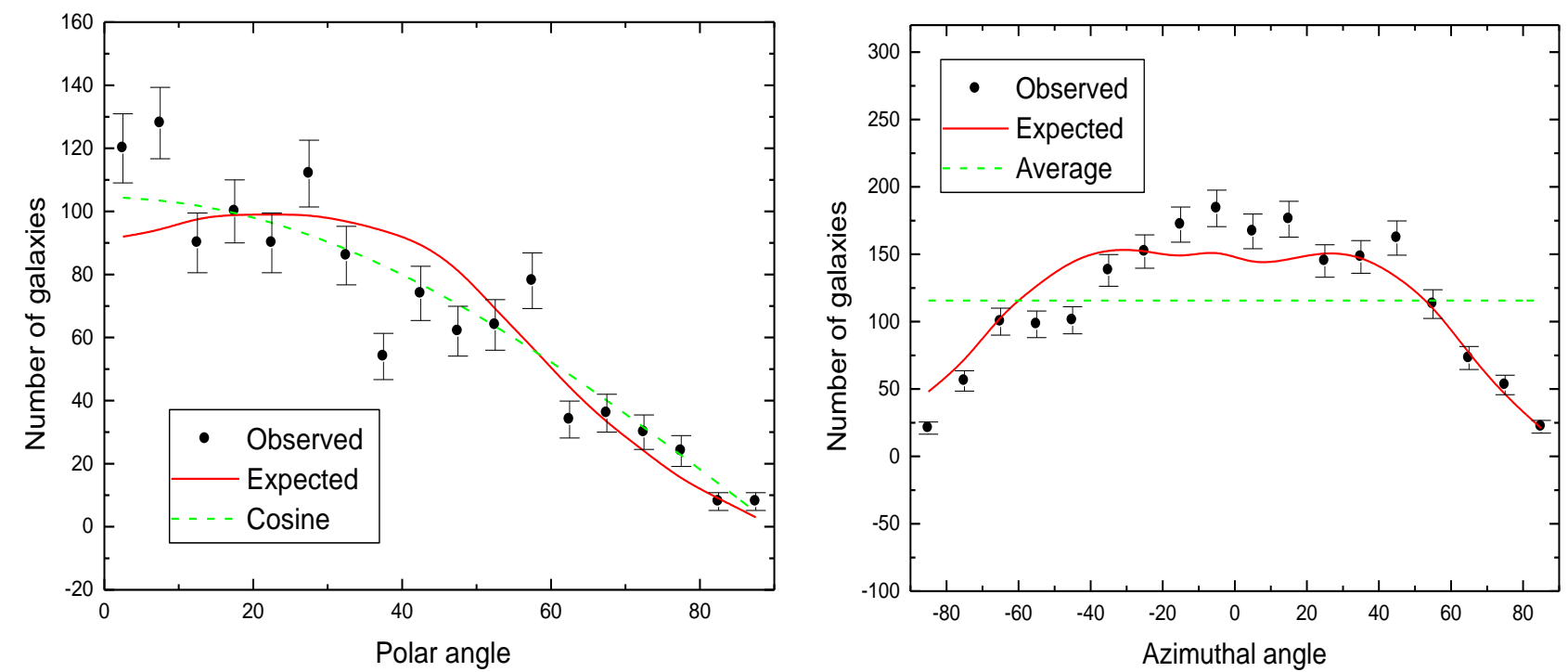

Fig. 1: The polar $(\theta)$ and azimuthal $(\phi)$ angle distributions of galaxies in the Supercluster $S[195+027+0022]$. The solid line represents the expected isotropic distributions. The dash lines represent the cosine and average distributions respectively. The solid circles with \pm 1 oerror bars are represent the observed distribution.

\section{CONCLUSION}

We have studied the preferred alignment of spin vector orientation of 1198 SDSS galaxies of Supercluster $\mathrm{S}[195+027+0022]$ that have redshift in the range 0.07 to 0.09 . We used the method proposed by Flin \& Godlowski [12] in order to compute two-dimensional data to three dimensional galaxy rotation axis (polar \& azimuthal angles). We have carried out random simulation by creating $10^{7}$ virtual galaxies and adopting the method proposed by Aryal \& Saurer [7] in order to find theoretical distribution of galaxy rotation axes. We have compared the differences between theoretical distributions and observed distributions using three statistics, namely chi-square, auto-correlation and the Fourier. The distribution of spin vector and spin vector projections of total SDSS galaxies that have redshift in the range 0.07 to 0.09 are found to be random in all samples, our results support Hierarchy model [22].

However, a local effect that causes the humps and dips in the angular momentum distribution is observed in different samples. In the deep field, density fluctuation is expected and observed in the local scale. Existence of Superclusters in the region of interest cannot be ruled out. We have used equatorial system as a physical reference in order to study non-random effects concerning galaxy orientation. Hierarchy model predicts that the choice of co-ordinate system do not alter preferred alignments.

\section{REFERENCES}

[1] Aryal B., Yadav S. N. \& Saurer W., Bulletin of Astron. Astron. Soc. Ind. (BASI), 40, 65 (2012).

[2] Aryal, B., Paudel, S., Saurer, W.: Astronom. Astrophys. 479, 397 (2008).

[3] Aryal, B., Paudel, S., Saurer, W.; MNRAS, 479, 397 (2007).

[4] Aryal, B., Saurer, W.: Monthly Notices Royal Astron. Soc. 366, 438 (2006).

[5] Aryal, B., Saurer, W.: Astronom. Astrophys. 432, 841 (2005).

[6] Aryal, B., Saurer, W.: Astronom. Astrophys. 425, 871 (2004).

[7] Aryal, B., Saurer, W.: Astronom. Astrophys. lett. 364, L97 (2000).

[8] Colless M., et. al., Monthly Notices Royal Astron. Soc. 328, 1039 (2001).

[9] Doroshkevich A. G., Shandarin S. F.: Monthly Notices Royal Astron. Soc. 184, 643 (1978).

[10] Doroshkevich A. G., Astrophysics, 6, 320 (1970).

[11] Doroshkevich A. G.: Astrophysical Journ. 14, L11 (1973).

[12] Flin P., Godlowski W.: Monthly Notices Royal Astron. Soc. 222, 525 (1986).

[13] Flin P.: Monthly Notices Royal Astron. Soc.325, 49 (2001). 
[14] Gamow G., Phys. Rev. 86, 251 (1952).

[15] Gamow \& Teller, Phys. Rev., 55, 654, (1939).

[16] Godlowski, W.: Monthly Notices Royal Astron. Soc. 265, 874 (1993).

[17] Godlowski, W.: Monthly Notices Royal Astron. Soc. 271, 19 (1994).

[18] Holmberg, E., LundAstro. Series-II, 117, 3 (1946).

[19] Heidmann J.,Heidmann N., de Vaucouleurs G.:Monthly Notices Royal Astron. Soc.75, 85 (1971).
[20] Ozernoy L. M., in Longair M. S., Einasto J., eds, Proc. IAU Symp. 79, The Large Scale Structure of the Universe. Reidel, Dordrecht (1978).

[21] Peebles P. J. E.: Astrophysical Journ .142, 1317 (1967)

[22] Peebles P. J. E.: Astrophysical Journ.155, 393 (1969).

[23] Stein R.: Astron. \&Astrophys.35, 17 (1974).

[24] von Weizsaker, The Astrophysical Journal(1951).

[25] York, D.G., Adelman, J., Anderson, J.E.: The Sloan Digital Sky Survey: Technical Summary (2000). 